\title{
Pacific
}

Journal of

Mathematics

\section{EMBEDDED CONSTANT-CURVATURE CURVES ON CONVEX SURFACES}

HAROLd Rosenberg AND MATTHIAS SchNEIDER 


\title{
EMBEDDED CONSTANT-CURVATURE CURVES ON CONVEX SURFACES
}

\author{
Harold Rosenberg AND Matthias Schneider
}

\begin{abstract}
We prove the existence of embedded closed constant-curvature curves on convex surfaces.
\end{abstract}

\section{Introduction}

Let $\left(S^{2}, g\right)$ be a two-dimensional oriented sphere with a smooth Riemannian metric $g$. We prove existence results for closed embedded curves with prescribed geodesic curvature in $\left(S^{2}, g\right)$, when the Gauss curvature $K_{g}$ of the metric $g$ is positive. In particular, we study the existence of closed embedded constant-curvature curves on strictly convex spheres.

Let $c: S^{2} \rightarrow \mathbb{R}$ be a smooth positive function. We consider the following equation for curves $\gamma$ on $S^{2}$ :

$$
D_{t, g} \dot{\gamma}(t)=|\dot{\gamma}(t)|_{g} c(\gamma(t)) J_{g}(\gamma(t)) \dot{\gamma}(t),
$$

where $D_{t, g}$ is the covariant derivative with respect to $g$, and $J_{g}(x)$ is the rotation by $\pi / 2$ in $T_{x} S^{2}$ with respect to $g$ and the given orientation. Solutions $\gamma$ to Equation (1-1) are constant-speed curves with geodesic curvature $c_{g}(\gamma, t)$ given by $c(\gamma(t))$. Besides the geometric interpretation, (1-1) describes the motion of a charged particle on $\left(S^{2}, g\right)$ in a magnetic field with magnetic form $c \mu_{g}$, where $\mu_{g}$ denotes the volume form of $g$ [Arnold 1986; Novikov 1982; Ginzburg 1996].

By [Schneider 2011b; Taimanov 1992], closed embedded solutions to (1-1) exist if the curvature function $c$ is large enough, depending on the metric $g$. When $g$ is $\frac{1}{4}$-pinched, that is, $\sup K_{g}<4 \inf K_{g}$, then there are embedded closed solutions of (1-1) for every positive function $c$ [Schneider 2011b; Rosenberg and Smith 2010]. It is conjectured [Novikov 1982, §5; Rosenberg and Smith 2010] that this remains true for an arbitrary metric $g$ on $S^{2}$. If $K_{g}$ and $c$ are positive, then by [Rosenberg and Smith 2010; Robadey 2001; Schneider 2011a] there are always Alexandrovembedded, closed solutions to (1-1), that is, curves that bound an immersed disc.

MSC2010: primary 53C42; secondary 37J45, 58E10.

Keywords: prescribed geodesic curvature. 
We show that on strictly convex spheres, that is, $K_{g}>0$, there are closed embedded solutions to (1-1) if the curvature function is small enough, depending on the metric $g$. In particular, we show:

Theorem 1.1. Suppose $\left(S^{2}, g\right)$ has positive Gauss curvature. Then there exists an $\varepsilon_{0}>0$ such that for all $0<c \leq \varepsilon_{0}$ there are two embedded closed curves with constant geodesic curvature $c$.

Hence, on strictly convex spheres, there are closed, embedded constant-curvature curves for large and small values of $c>0$. We conjecture that this remains true for all $c>0$ and any metric on $S^{2}$.

We use the degree theory developed in [Schneider 2011b] to prove our existence result. The required compactness results are given in Section 2. The a priori estimates follow from Reilly's formula [1977]. The fact that a geodesic cannot touch itself continues to hold for solutions to (1-1) when the geodesic curvature is close to zero. This allows us to carry out the degree argument within the class of embedded curves. The existence result is given in Section 3.

\section{The a priori estimate}

Lemma 2.1. Suppose $\left(S^{2}, g\right)$ has positive Gauss curvature $K_{g}$ and $\gamma \in C^{2}\left(S^{1}, S^{2}\right)$ is an (Alexandrov) embedded curve with nonnegative geodesic curvature. Then the length $L(\gamma)$ of $\gamma$ is bounded by

$$
L(\gamma) \leq 2 \pi \sqrt{2}\left(\inf _{S^{2}} K_{g}\right)^{-1 / 2} .
$$

Proof. As in [Choi and Wang 1983], where area bounds for embedded compact minimal surfaces in $S^{3}$ are given, we use Reilly's formula [1977]: Let $(M, g)$ be a compact Riemannian manifold with boundary $\partial M, f \in C^{\infty}(M), z=\left.f\right|_{\partial M}$ and $u=\partial f / \partial n$ on $\partial M$, where $n$ denotes the outer normal. Then

$$
\begin{aligned}
\int_{M}(\bar{\Delta} f)^{2}-\left|\bar{\nabla}^{2} f\right|^{2} & \\
= & \int_{M} \operatorname{Ric}(\bar{\nabla} f, \bar{\nabla} f)+\int_{\partial M}(\Delta z+H u) u-\langle\nabla z, \nabla u\rangle+\Pi(\nabla z, \nabla z),
\end{aligned}
$$

where we denote by $\bar{\Delta}, \Delta$ and $\bar{\nabla}, \nabla$ the Laplacians and covariant derivatives on $M$ and $\partial M$ respectively; $H$ is the mean curvature and $\Pi$ is the second fundamental form of $\partial M$.

If the curve $\gamma$ is embedded or Alexandrov-embedded, then we may assume that we are in the above situation with $\partial M=\gamma$.

We take $z$ an eigenfunction of $\lambda_{1}$ the first nontrivial eigenvalue on $\partial M$,

$$
\Delta z+\lambda_{1} z=0 \text { on } \partial M
$$


and $f$ its harmonic extension to $M$. In dimension two, (2-1) leads to

$$
\int_{M}(\bar{\Delta} f)^{2}-\left|\bar{\nabla}^{2} f\right|^{2}=\int_{M} K_{g}|\bar{\nabla} f|^{2}+\int_{\partial M} \Delta z u+c u^{2}-\langle\nabla z, \nabla u\rangle+c|\nabla z|^{2},
$$

where $c$ is the geodesic curvature of $\partial M$ and $K_{g}$ denotes the Gauss curvature of $M$. Using the facts that the geodesic curvature $c$ of $\partial M$ is nonnegative, $f$ is harmonic, and $z$ is an eigenfunction, we obtain

$$
0 \geq\left(\inf _{M} K_{g}\right) \int_{M}|\bar{\nabla} f|^{2}-2 \lambda_{1} \int_{\partial M} z u .
$$

Integrating by parts again, we see

$$
\int_{\partial M} z u=\int_{M}|\bar{\nabla} f|^{2}+f \bar{\Delta} f=\int_{M}|\bar{\nabla} f|^{2} .
$$

Since $z$ is a nontrivial eigenfunction, $f$ is nonconstant and we arrive at

$$
\left(\inf _{M} K_{g}\right) \leq 2 \lambda_{1} .
$$

The first nontrivial eigenvalue $\lambda_{1}$ depends only on the length $L(\partial \Omega)$ of $\partial M$ and is given by

$$
\lambda_{1}=\frac{4 \pi^{2}}{L(\partial \Omega)^{2}} .
$$

Lemma 2.2. Let $\left(\gamma_{n}\right)$ be a sequence of simple closed curves that converge in $C^{2}\left(S^{1}, S^{2}\right)$ to a nonconstant closed geodesic $\gamma$ in $\left(S^{2}, g\right)$. Then $\gamma$ is also simple.

Proof. To obtain a contradiction, assume that there are $\theta_{1} \neq \theta_{2}$ in $S^{1}=\mathbb{R} / \mathbb{Z}$ such that $\gamma\left(\theta_{1}\right)=\gamma\left(\theta_{2}\right)$. Since $\gamma$ is a limit of simple curves and $|\dot{\gamma}| \equiv$ const, we have

$$
\dot{\gamma}\left(\theta_{1}\right)= \pm \dot{\gamma}\left(\theta_{2}\right) \text {. }
$$

From the uniqueness of geodesics, we have for $t \in S^{1}$

$$
\gamma(t)=\gamma\left( \pm\left(t-\theta_{1}\right)+\theta_{2}\right) .
$$

Setting $t=\left(\theta_{1}+\theta_{2}\right) / 2$, we find that

$$
\gamma(t)=\gamma\left(t-\theta_{1}+\theta_{2}\right) .
$$

Consequently, $\gamma$ is an $n$-fold covering of a simple geodesic for some $n \geq 2$. From the stability of the winding number, we get a contradiction.

We denote by $g_{\text {can }}$ the standard round metric on $S^{2}$ with curvature $K_{g_{\text {can }}} \equiv 1$. We fix a function $\varphi \in C^{\infty}\left(S^{2}, \mathbb{R}\right)$ and a conformal metric

$$
g=e^{\varphi} g_{\mathrm{can}}
$$


on $S^{2}$ with positive Gauss curvature $K_{g}>0$. We consider the family of metrics $\left\{g_{t}: t \in[0,1]\right\}$ defined by

$$
g_{t}:=e^{t \varphi} g_{\text {can }} .
$$

Then the Gauss curvature $K_{g_{t}}$ of the metric $g_{t}$ satisfies, for some $K_{0}>0$,

$$
\begin{aligned}
K_{g_{t}} & =e^{-t \varphi}\left(-t \Delta_{g_{\mathrm{can}}}(\varphi)+2\right) \\
& =e^{-t \varphi}\left(-t\left(2-K_{g} e^{\varphi}\right)+2\right) \geq K_{0},
\end{aligned}
$$

because $K_{g}$ is positive.

Lemma 2.3. Suppose $c: S^{2} \rightarrow \mathbb{R}$ is a nonnegative smooth function. For $r \in[0,1]$, we define the set of closed curves $\mathcal{M}_{r}$ by $M_{r}:=\left\{\gamma \in C^{2}\left(S^{1}, S^{2}\right): \gamma\right.$ is embedded, $|\dot{\gamma}|_{g} \equiv$ const,

and there exists $(t, s) \in[0,1] \times[0, r]: c_{g_{t}}(\gamma, \theta)=s c(\gamma(\theta))$ for all $\left.\theta \in S^{1}\right\}$, where $c_{g_{t}}(\gamma, \cdot)$ denotes the geodesic curvature of $\gamma$ with respect to $g_{t}$.

Then there is $\varepsilon_{0}>0$ such that $\mathcal{M}_{\varepsilon_{0}}$ is compact. Moreover, $\varepsilon_{0}>0$ may be chosen uniformly with respect to $\|c\|_{\infty}$.

Proof. Let $\left(\gamma_{n}\right)_{n \in \mathbb{N}}$ be a sequence in $\mu_{r}$ for some $r>0$. By Lemma 2.1 and (1-1), we get a uniform bound in $C^{3}\left(S^{1}, S^{2}\right)$, and from the Gauss-Bonnet formula, the length of $\gamma_{n}$ is bounded below; and in both cases the bounds are uniform with respect to $r$. Since the metrics $\left\{g_{t}: t \in[0,1]\right\}$ are uniformly equivalent, there is $C_{0}>0$ such that we have for all $t \in[0,1]$

$$
\left|\dot{\gamma}_{n}\right|_{g_{t}}>\left(C_{0}\right)^{-1} \text { and }\left\|\gamma_{n}\right\|_{C^{3}\left(S^{1}, S^{2}\right), g_{t}}<C_{0} .
$$

Up to a subsequence we may assume $\left(t_{n}, s_{n}\right) \rightarrow(t, s) \in[0,1] \times[0, r]$,

$$
\gamma_{n} \rightarrow \gamma \text { in } C^{2}\left(S^{1}, S^{2}\right)
$$

where $|\dot{\gamma}|_{g_{t}} \equiv$ const and

$$
c_{g_{t}}(\gamma, \theta)=s c(\gamma(\theta)) \text { for all } \theta \in S^{1} .
$$

Thus, if $\mu_{r}$ is not compact, there is $(t, s) \in[0,1] \times[0, r]$ and $\gamma_{r} \in C^{2}\left(S^{1}, S^{2}\right)$ satisfying $|\dot{\gamma}|_{g_{t}} \equiv$ const and (2-3), which is not embedded, but a limit of embedded curves in $M_{r}$. Thus there are $\theta_{1}, \theta_{2} \in S^{1}$, such that $\theta_{1} \neq \theta_{2}$ and $\gamma_{r}\left(\theta_{1}\right)=\gamma_{r}\left(\theta_{2}\right)$. From (2-2) we deduce that there is $\delta>0$ independent of $r$, such that

$$
\delta \leq\left|\theta_{1}-\theta_{2}\right| \leq 1-\delta .
$$

Hence for any $n \in \mathbb{N}$ there is $\gamma_{n} \in M_{r}$ such that

$$
\operatorname{dist}\left(\gamma_{n}\left(\theta_{1}\right), \gamma_{n}\left(\theta_{2}\right)\right) \leq \frac{1}{n}
$$


To obtain a contradiction, assume there is $\left(r_{n}\right)$ converging to 0 such that $M_{r_{n}}$ is not compact. Then for any $n \in \mathbb{N}$, there are $\left(t_{n}, s_{n}\right) \in[0,1] \times\left[0, r_{n}\right], \theta_{1, n}, \theta_{2, n} \in S^{1}$, and $\gamma_{n} \in \mathcal{M}_{r_{n}}$ that satisfy (2-4) and (2-5). From the uniform bounds, going to a subsequence, we may assume that $\left(t_{n}, s_{n}, \gamma_{n}, \theta_{1, n}, \theta_{2, n}\right)$ converge to $\left(t, 0, \gamma, \theta_{1}, \theta_{2}\right)$, where $\theta_{1}$ and $\theta_{2}$ satisfy (2-4) and $\gamma$ is a closed nontrivial geodesic in $\left(S^{2}, g_{t}\right)$ satisfying $\gamma\left(\theta_{1}\right)=\gamma\left(\theta_{2}\right)$. This contradicts Lemma 2.2. Since all the above bounds are uniform with respect to $\|c\|_{\infty}$, the constant $\varepsilon_{0}>0$ may be chosen uniform with respect to $\|c\|_{\infty}$ as well.

\section{Existence results}

We follow [Schneider 2011b] and consider solutions to (2-3) as zeros of the vector field $X_{c, g}$ defined on the Sobolev space $H^{2,2}\left(S^{1}, S^{2}\right)$ as follows: For $\gamma \in$ $H^{2,2}\left(S^{1}, S^{2}\right)$, we let $X_{c, g}(\gamma)$ be the unique weak solution of

$$
\left(-D_{t, g}^{2}+1\right) X_{c, g}(\gamma)=-D_{t, g} \dot{\gamma}+|\dot{\gamma}|_{g} c(\gamma) J_{g}(\gamma) \dot{\gamma}
$$

in $T_{\gamma} H^{2,2}\left(S^{1}, S^{2}\right)$

Solutions to (2-3), or equivalently, zeros of $X_{c, g}$, are invariant under a circle action: For $\theta \in S^{1}=\mathbb{R} / \mathbb{Z}$ and $\gamma \in H^{2,2}\left(S^{1}, S^{2}\right)$, we define $\theta * \gamma \in H^{2,2}\left(S^{1}, S^{2}\right)$ by

$$
\theta * \gamma(t)=\gamma(t+\theta) .
$$

Thus, any solution gives rise to an $S^{1}$-orbit of solutions, and we say that two solutions $\gamma_{1}$ and $\gamma_{2}$ are (geometrically) distinct if $S^{1} * \gamma_{1} \neq S^{1} * \gamma_{2}$.

We denote by $M \subset H^{2,2}\left(S^{1}, S^{2}\right)$ the set

$$
M:=\left\{\gamma \in H^{2,2}\left(S^{1}, S^{2}\right): \dot{\gamma}(\theta) \neq 0 \text { for all } \theta \in S^{1} \text { and } \gamma \text { is embedded }\right\} .
$$

In [Schneider 2011b], an integer-valued $S^{1}$-degree $\chi_{S^{1}}\left(X_{c, g}, M\right)$ is introduced. The $S^{1}$-degree is defined whenever $X_{c, g}$ is proper in $M$, that is, the set

$$
\left\{\gamma \in M: X_{c, g}(\gamma)=0\right\}
$$

is compact, and does not change under homotopies in the class of proper vector fields.

Theorem 3.1. Suppose $\left(S^{2}, g\right)$ has positive Gauss curvature. Then there is $\varepsilon_{0}>0$ such that for all smooth functions $c: S^{2} \rightarrow \mathbb{R}$ satisfying $0<c \leq \varepsilon_{0}$, there are two embedded geometrically distinct closed curves that solve Equation (1-1).

Proof. From the uniformization theorem up to isometries, we may assume without loss of generality that

$$
g=e^{\varphi} g_{\text {can }},
$$

where $\varphi \in C^{\infty}\left(S^{2}, \mathbb{R}\right)$ and $g_{\text {can }}$ denotes the standard round metric on $S^{2}$. 
We consider the set of metrics $\left\{g_{t}: t \in[0,1]\right\}$ defined by

$$
g_{t}:=e^{t \varphi} g_{\text {can }} .
$$

From Lemma 2.3, there is $\varepsilon_{0}>0$ such that the set

$$
\left\{\gamma \in M: X_{c, g_{t}}(\gamma)=0 \text { for some } t \in[0,1]\right\}
$$

is compact for all functions $c$ with $0<c \leq \varepsilon_{0}$. Consequently,

$$
[0,1] \ni t \mapsto X_{c, g_{t}}
$$

is a homotopy of proper vector fields. From [Schneider 2011b], we have

$$
-2=\chi_{S^{1}}\left(X_{c, g_{\text {can }}}, M\right),
$$

such that the homotopy invariance leads to

$$
\chi_{S^{1}}\left(X_{c, g}, M\right)=-2 .
$$

Since the local degree of an isolated zero orbit is greater than or equal to -1 by [Schneider 2011b, Lemma 4.1], there are at least two geometrically distinct solutions to (1-1). This gives the claim.

\section{References}

[Arnold 1986] V. I. Arnold, “The first steps of symplectic topology”, Uspekhi Mat. Nauk 41:6(252) (1986), 3-18, 229. MR 890489 (89d:58034) Zbl 0618.58021

[Choi and Wang 1983] H. I. Choi and A. N. Wang, "A first eigenvalue estimate for minimal hypersurfaces”, J. Differential Geom. 18:3 (1983), 559-562. MR 723817 (85d:53028) Zbl 0523.53055

[Ginzburg 1996] V. L. Ginzburg, "On closed trajectories of a charge in a magnetic field. An application of symplectic geometry”, pp. 131-148 in Contact and symplectic geometry (Cambridge, 1994), Publ. Newton Inst. 8, Cambridge Univ. Press, 1996. MR 1432462 (97j:58128) Zbl 0873.58034

[Novikov 1982] S. P. Novikov, "The Hamiltonian formalism and a many-valued analogue of Morse theory", Uspekhi Mat. Nauk 37:5(227) (1982), 3-49, 248. MR 676612 (84h:58032) Zbl 0571. 58011

[Reilly 1977] R. C. Reilly, "Applications of the Hessian operator in a Riemannian manifold", Indiana Univ. Math. J. 26:3 (1977), 459-472. MR 0474149 (57 \#13799) Zbl 0391.53019

[Robadey 2001] A. Robadey, Autour des géodésiques fermées simples sur la sphère, Master's thesis, Université Paris VII, 2001.

[Rosenberg and Smith 2010] H. Rosenberg and G. Smith, "Degree Theory of Immersed Hypersurfaces", preprint, 2010. arXiv 1010.1879

[Schneider 2011a] M. Schneider, "Alexandrov embedded closed magnetic geodesics on $S^{2}$ ", preprint, 2011. To appear in Ergodic Theory Dynam. Systems. arXiv 0903.1128

[Schneider 2011b] M. Schneider, "Closed Magnetic Geodesics on $S^{2}$ ", J. Differential Geom. 87:2 (2011), 343-388. MR 2788659

[Taimanov 1992] I. A. Taimanov, "Closed extremals on two-dimensional manifolds", Uspekhi Mat. Nauk 47:2(284) (1992), 143-185, 223. MR 1185286 (93k:58050) Zbl 0791.58026 
Received June 8, 2011.

HAROLd Rosenberg

Instituto de Matemática Pura e Aplicada

110 ESTRADA DONA CASTORINA

22460-320 RIO DE JANEIRO

BRAZIL

rosen@impa.br

MATTHias SCHNEIDER

MATHEMATISCHES INSTITUT

RUPRECHT-KARLS-UNIVERSITÄT

IM NEUENHEIMER FELD 288

D-69120 HEIDELBERG

GERMANY

mschneid@mathi.uni-heidelberg.de 


\title{
PACIFIC JOURNAL OF MATHEMATICS
}

\author{
http://www.pjmath.org \\ Founded in 1951 by
}

E. F. Beckenbach (1906-1982) and F. Wolf (1904-1989)

\section{EDITORS}

V. S. Varadarajan (Managing Editor)

Department of Mathematics

University of California

Los Angeles, CA 90095-1555

pacific@math.ucla.edu

Vyjayanthi Chari

Department of Mathematics

University of California

Riverside, CA 92521-0135

chari@math.ucr.edu

\section{Robert Finn}

Department of Mathematics Stanford University

Stanford, CA 94305-2125

finn@math.stanford.edu

Kefeng Liu

Department of Mathematics

University of California

Los Angeles, CA 90095-1555

liu@math.ucla.edu
Darren Long

Department of Mathematics

University of California

Santa Barbara, CA 93106-3080

long@math.ucsb.edu

Jiang-Hua Lu

Department of Mathematics

The University of Hong Kong

Pokfulam Rd., Hong Kong jhlu@maths.hku.hk

Alexander Merkurjev

Department of Mathematics University of California

Los Angeles, CA 90095-1555 merkurev@math.ucla.edu
Sorin Popa

Department of Mathematics

University of California

Los Angeles, CA 90095-1555

popa@math.ucla.edu

Jie Qing

Department of Mathematics

University of California

Santa Cruz, CA 95064

qing@ cats.ucsc.edu

Jonathan Rogawski

Department of Mathematics

University of California

Los Angeles, CA 90095-1555

jonr@math.ucla.edu

\section{PRODUCTION}

pacific@math.berkeley.edu

Silvio Levy, Scientific Editor Matthew Cargo, Senior Production Editor

ACADEMIA SINICA, TAIPEI

CALIFORNIA INST. OF TECHNOLOGY

INST. DE MATEMÁTICA PURA E APLICADA

KEIO UNIVERSITY

MATH. SCIENCES RESEARCH INSTITUTE

NEW MEXICO STATE UNIV.

OREGON STATE UNIV.

\section{SUPPORTING INSTITUTIONS}

STANFORD UNIVERSITY
UNIV. OF BRITISH COLUMBIA
UNIV. OF CALIFORNIA, BERKELEY
UNIV. OF CALIFORNIA, DAVIS
UNIV. OF CALIFORNIA, LOS ANGELES
UNIV. OF CALIFORNIA, RIVERSIDE
UNIV. OF CALIFORNIA, SAN DIEGO
UNIV. OF CALIF., SANTA BARBARA

UNIV. OF CALIF., SANTA CRUZ

UNIV. OF MONTANA

UNIV. OF OREGON

UNIV. OF SOUTHERN CALIFORNIA

UNIV. OF UTAH

UNIV. OF WASHINGTON

WASHINGTON STATE UNIVERSITY

These supporting institutions contribute to the cost of publication of this Journal, but they are not owners or publishers and have no responsibility for its contents or policies.

See inside back cover or www.pjmath.org for submission instructions.

The subscription price for 2011 is US \$420/year for the electronic version, and \$485/year for print and electronic.

Subscriptions, requests for back issues from the last three years and changes of subscribers address should be sent to Pacific Journal of Mathematics, P.O. Box 4163, Berkeley, CA 94704-0163, U.S.A. Prior back issues are obtainable from Periodicals Service Company, 11 Main Street, Germantown, NY 12526-5635. The Pacific Journal of Mathematics is indexed by Mathematical Reviews, Zentralblatt MATH, PASCAL CNRS Index, Referativnyi Zhurnal, Current Mathematical Publications and the Science Citation Index.

The Pacific Journal of Mathematics (ISSN 0030-8730) at the University of California, c/o Department of Mathematics, 969 Evans Hall, Berkeley, CA 94720-3840, is published monthly except July and August. Periodical rate postage paid at Berkeley, CA 94704, and additional mailing offices. POSTMASTER: send address changes to Pacific Journal of Mathematics, P.O. Box 4163, Berkeley, CA 94704-0163.

PJM peer review and production are managed by EditFLOW ${ }^{\mathrm{TM}}$ from Mathematical Sciences Publishers.

PUBLISHED BY PACIFIC JOURNAL OF MATHEMATICS

at the University of California, Berkeley 94720-3840

A NON-PROFIT CORPORATION

Typeset in IATEX

Copyright $(2011$ by Pacific Journal of Mathematics 


\title{
PACIFIC JOURNAL OF MATHEMATICS
}

\author{
Volume $253 \quad$ No. $1 \quad$ September 2011
}

Singularities of the projective dual variety

ROLAND ABUAF

Eigenvalue estimates for hypersurfaces in $\mathbb{H}^{m} \times \mathbb{R}$ and applications

Pierre BÉRARD, Philippe CASTILlon and Marcos CaVAlCante

Conformal Invariants associated to a measure: Conformally covariant operators

Sun-Yung A. Chang, Matthew J. Gursky and Paul Yang

Compact symmetric spaces, triangular factorization, and Cayley coordinates

DEREK HABERMAS

Automorphisms of the three-torus preserving a genus-three Heegaard splitting

JESSE JOHNSON

The rationality problem for purely monomial group actions

HiDETAKA KiTAYAMA

On a Neumann problem with $p$-Laplacian and noncoercive resonant nonlinearity

Salvatore A. Marano and Nikolaos S. Papageorgiou

Minimal ramification in nilpotent extensions

NADYA MARKIN and STEPHEN V. ULLOM

Regularity of weakly harmonic maps from a Finsler surface into an $n$-sphere

XIAOHUAN MO and LIANG ZHAO

On the sum of powered distances to certain sets of points on the circle

NikOlai NikOlOV and RAFAEL RAFAilov

Formal geometric quantization II

PAUL-ÉMILE PARADAN

Embedded constant-curvature curves on convex surfaces

HAROLD ROSENBERG and MATTHIAS SCHNEIDER

A topological construction for all two-row Springer varieties 